\title{
On the node reliability of Harary graphs: an analysis through real-world topologies
}

This paper was downloaded from TechRxiv (https://www.techrxiv.org).

\section{LICENSE}

CC BY 4.0

SUBMISSION DATE / POSTED DATE

$19-11-2021 / 24-11-2021$

\section{CITATION}

de Lima, Leonardo (2021): On the node reliability of Harary graphs: an analysis through real-world topologies. TechRxiv. Preprint. https://doi.org/10.36227/techrxiv.17049419.v1

DOI 


\title{
On the node reliability of Harary graphs: an analysis through real-world topologies
}

\author{
Gloria Ney, CEFET-RJ, Leonardo de Lima, UFPR, Carla Oliveira, ENCE/IBGE, Jose E. Pecora Junior, UFPR, \\ and Marcia Paiva, UFES
}

\begin{abstract}
The literature on network reliability shows that Harary networks are designed so that the link reliability is maximum in many cases. The following question: "what are the best topology networks in maximizing node reliability?" is still open. In this paper, we performed computational experiments with eleven real-world networks and their corresponding Harary graphs. The node reliability of both sets of networks was computed. Computational results point out that the Harary network has a topology with high node reliability if compared to the real-world networks studied.
\end{abstract}

Index Terms-Harary graph, Network, node reliability.

\section{INTRODUCTION}

The reliability of a network is defined as the probability that the network remains connected even after removing a subset of links or nodes. A network is more reliable if it has less probability of becoming disconnected after some random failures. The problem of determining the most reliable networks often appears in the literature according to the following four types: (A) the nodes are reliable and only links may fail; (B) the links are reliable, and only the nodes may fail; (C) links and nodes may fail independently; (D) an optimization problem is described where an objective function is optimized under some constraints involving a reliability measure. Approaches of types (A) and (B) are related to a reliability measure that depends on the link and node connectivity parameters and the cardinality of the link cut set or node cut set. Approaches of type (C) are more related to Monte Carlo simulation models, and in (D), dynamic and heuristic methods are amongst the most used to address the problem. Some references about that subjects are [1], [4], [6], [7], [8], [14], [20], [21], [22], [24], [25]. In designing a communication system, it is desirable that it still works in case of disruption or failure of some of its nodes [18]. Here, we address the problem of type (B). In this context, since Harary graphs [11] are very well-known in the literature as the ones with the largest link and node connectivity under certain conditions to the order and number of links, such class of graphs plays an important role in our

G. M. A. Ney is at Centro Federal de Educação Tecnologica Celso Suckow da Fonseca, Rio de Janeiro, RJ 20271-204 BRA e-mail: gloria.alves@aluno.cefet-rj.br .

L. S. de Lima is at Universidade Federal do Parana, Curitiba, PR 80060-000 BRA e-mail: leonardo.delima@ufpr.br

C. Oliveira is at ENCE/IBGE, Rio de Janeiro, RJ 20271-204 BRA e-mail: carla.oliveira@ibge.gov.br

J. E. Pecora, Jr. is at Universidade Federal do Parana, Curitiba, PR 80060000 BRA e-mail: pecora@ufpr.br

M. Paiva is at Universidade Federal do Espírito Santo, Vitória, ES, BRA e-mail: pecora@ufpr.br study. It turns out that some Harary topologies are also the most reliable network topologies in the context of the problem of type (A), [1]. However, to the best of our knowledge, there is no paper showing how good is the node reliability of a Harary network topology compared to any network of the same order and size. In this paper, computational experiments are performed over a dataset of eleven real-world optical backbone networks. We computed the node reliability of each real-world network topology and compared it to the node reliability of the corresponding Harary network. Besides, we propose a simplified formula to compute a network's node reliability, which shows to be useful for networks with a high number of nodes and links.

\section{NETWORK RELIABILITY}

A physical network topology can be modelled as a graph $G$ with node set $V(G)$ of cardinality $|V|=N$ and link set $E(G)$ of cardinality $|E|=L$. A set of links $S \subseteq E(G)$ is a link cut set if $G \backslash S$ is disconnected, i.e., if the removal of $S$ from $G$ disconnects the graph $G$. The link (resp. node) connectivity of a graph $G, k^{\prime}(G)$ (resp. $k(G)$ ), is the size of the smallest link (resp. node) cutset of G. For given $N$ and $k$, Harary [11] shows a procedure to generate a graph $H$ on $N$ nodes and minimum number of links $L$ that maximizes the link and node connectivity. Those graphs are called Harary graphs, [11], and such graphs satisfy the following equality:

$$
k(H)=k^{\prime}(H)=\left\lfloor\frac{2 L}{N}\right\rfloor .
$$

In [17], the authors present a variation of Hakimi's algorithm, [10], that builds Harary graphs from $N$ and $L$ given as inputs. The goal of the Hakimi's algorithm is to share the links between $N$ nodes in order to have a graph as wellbalanced as possible. It uses the classic Euclidean division procedure to distribute links between nodes by $L=q N+$ $r, 0 \leq q<N$ and $q=\left\lfloor\frac{L}{N}\right\rfloor$. Also, the algorithm considers the distances of nodes labeled as $i$ and $j$ as $|j-i|_{N}=$ $\min (j-i) \bmod n,(i-j) \bmod n$ according to Algorithm 1 . For instance, we consider the real-world optical backbone CESNET network with $N=12$ nodes and $L=19$ links, which is presented in Figure 1 and its corresponding Harary graph obtained by Algorithm 1 in Figure 2. It is proved, [6], that the Harary graph of Figure 2 has higher link reliability than the one of Figure 1. We are interested to observe what happens to the node reliability of real networks compared to the Harary topology. Assuming that links are perfect and nodes may fail randomly and independently at probability $1-p$ 

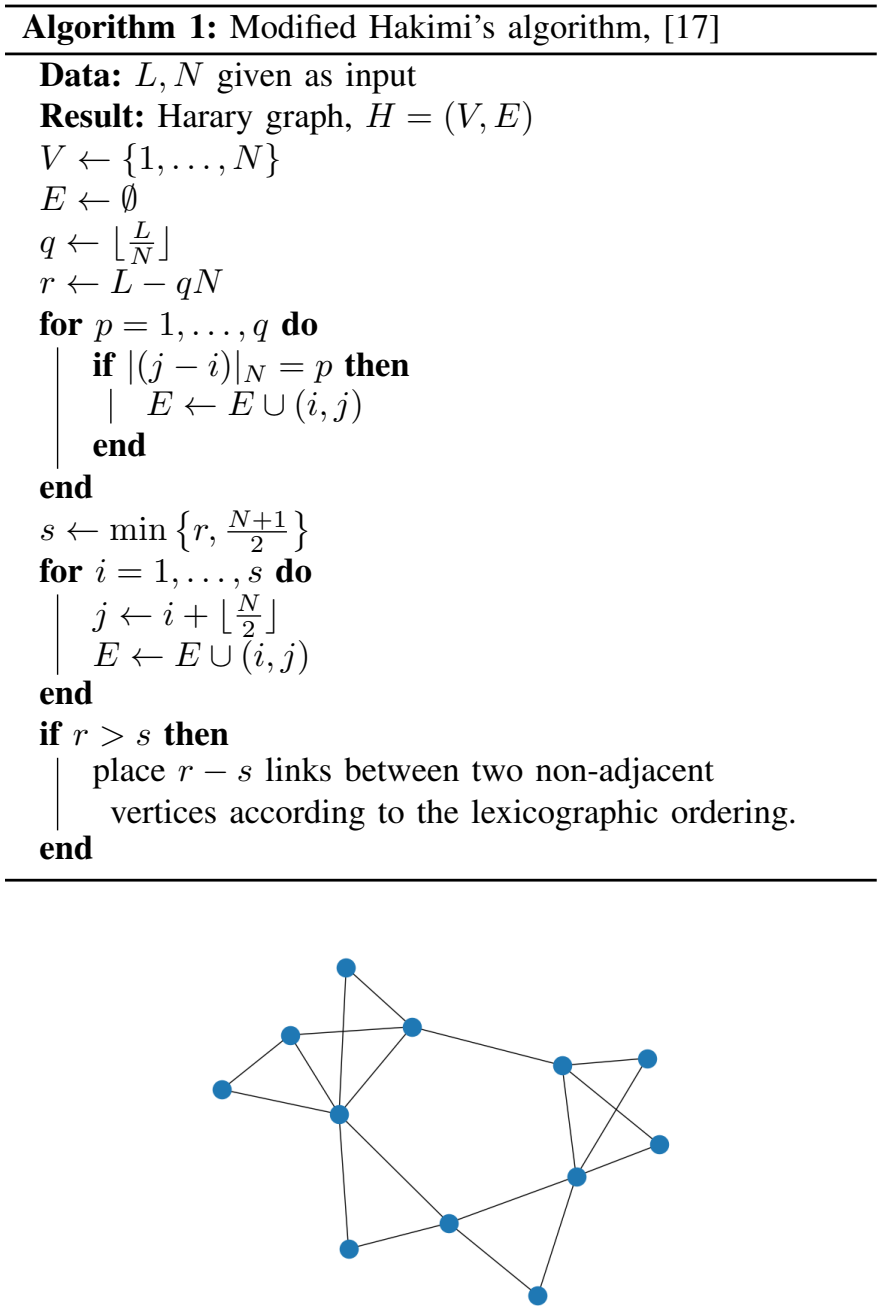

Fig. 1: CESNET network $G$

such that $p \in(0,1)$, the network reliability with node failures is defined by Equation (1):

$$
R(G, p)=\sum_{r=1}^{N} S_{r}^{\prime}(G) p^{r}(1-p)^{N-r},
$$

where $S_{r}^{\prime}(G)$ is the number of connected induced subgraphs of $G$ with $r$ nodes. Notice that in Equation (1), the parameter $p$ is the probability that a node does not fail. Let $\Omega(N, L)$ be the set of all connected networks with $N$ nodes and $L$ links. For a given $p$, a graph $G \in \Omega(N, L)$ is said to be the most reliable if $R(G, p) \geq R(H, p)$ for any $H \in \Omega(N, L)$. If $G \in \Omega(N, L)$ is the most reliable graph for any $p$, then $G$ is said to be uniformly best [9]. Notice that computing $S_{r}^{\prime}(G)$ for all $r$ is a very difficult computational task even for small values of $N$. Good approaches to $R(G, p)$ can be useful for large networks. Analogously, one can evaluate the graph reliability by considering that links may fail with equal and independent probability $p$ from Equation (2):

$$
R_{E}(G, p)=1-\sum_{i=k^{\prime}(G)}^{L} S_{i}(G) p^{i}(1-p)^{L-i}
$$

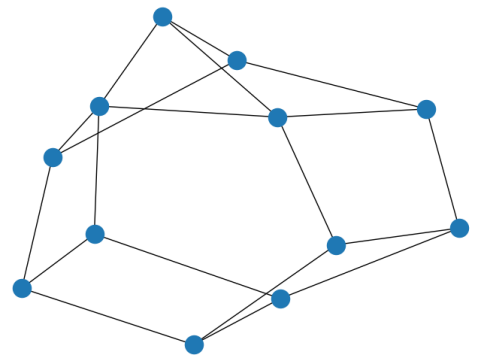

Fig. 2: Harary graph $H$ with $N=12$ nodes and $L=19$ links, obtained by Algorithm 1.

where $S_{i}(G)$ is the number of link cut sets of size $i$. In order to maximize $R_{E}(G, p), G$ should have maximum link connectivity, $k^{\prime}(G)$, and minimum link cut set, $S_{i}(G)$. In [6], under certain conditions, the authors proved that Harary graphs also have maximum reliability when we consider the problem of type (A), i.e., nodes are perfect, and links may fail. However, we are unaware of any paper relating network reliability under node failures to Harary graphs topology. This paper is about the investigation of such relations. Further, we propose simplifying the node reliability function based on our computational results with real-world networks.

\section{Methodology}

We selected 11 real-world optical backbone networks from the Topology Zoo [23] corresponding to some of the ones studied by [20]. These are Optical Transport Networks (OTNs), that carry large amounts of data at high rates through the DWDM (Dense Wavelength Division Multiplexing) technique. A single node failure in such a network may disrupt service on many optical links, leading to significant data and revenue losses. For that reason, it is worth investigating the node reliability of real-world optical backbone networks and to identify suitable network topologies regarding node reliability. In Table I each studied network is presented with its number of nodes, links, and average degree. The number of nodes of each network ranges from 12 to 32 , while the number of links ranges from 19 to 50. Table I is arranged in non-decreasing order according to the number of nodes. The fourth column is the average degree of each network which is computed by $2 L / N$. Our methodology consists of the following steps:

TABLE I: Real-world network topologies

\begin{tabular}{cccc}
\hline $\begin{array}{c}\text { Network } \\
\text { Topology }\end{array}$ & $\begin{array}{c}\text { Number of } \\
\text { Nodes }\end{array}$ & $\begin{array}{c}\text { Number of } \\
\text { Links }\end{array}$ & $\begin{array}{c}\text { Average } \\
\text { degree }\end{array}$ \\
\hline CESNET & 12 & 19 & 3.167 \\
ITALY & 14 & 29 & 4.143 \\
NSFNET & 14 & 21 & 3.000 \\
GARR-B & 16 & 27 & 3.375 \\
GERMANY & 17 & 26 & 3.059 \\
REDIRISNET & 17 & 28 & 3.294 \\
EON & 19 & 37 & 3.895 \\
COX & 24 & 40 & 3.333 \\
CERNET & 29 & 45 & 3.103 \\
IBN31 & 31 & 47 & 3.032 \\
GEANT2 & 32 & 50 & 3.125 \\
\hline
\end{tabular}

(1) For a fixed $p=0.05$, we compute $R(G, p)$ for all networks of Table I by computing $S_{1}^{\prime}(G), S_{2}^{\prime}(G), \ldots, S_{N}^{\prime}(G)$. 
(2) We generate the Harary graph, say $H$, with the same number of nodes and links of the networks of Table I and compute $R(H, p)$ for $p=0.05$ by obtaining $S_{1}^{\prime}(H), S_{2}^{\prime}(H), \ldots, S_{N}^{\prime}(H)$. All Harary graphs were obtained by applying Algorithm 1.

(3) We compare the results obtained in Steps (1) and (2) to the ones obtained for $R_{E}(G, p)$ and $R_{E}(H, p)$ available in [20].

\section{COMPUTATIONAL EXPERIMENTS}

All routines were implemented in Python 3.7 using a computer of $8 \mathrm{~Gb}$ RAM memory. We start presenting the node connectivity of each real-world network and the node connectivity of the corresponding Harary graph with the same number of links and nodes. Intuitively, we can think that the higher the node connectivity, the higher is the node reliability. The results are displayed in Table II. As theoretically expected, the Harary graph version of each network has higher node connectivity, except for the IBN31 network, where the node connectivities are equal. Table III presents the link and node

TABLE II: Networks' node connectivity

\begin{tabular}{ccc}
\hline Network & $k(G)$ & $k(H)$ \\
\hline CESNET & 2 & 3 \\
ITALY & 2 & 4 \\
NSFNET & 2 & 3 \\
GARR-B & 2 & 3 \\
GERMANY & 2 & 3 \\
REDIRISNET & 2 & 3 \\
EON & 2 & 3 \\
COX & 1 & 3 \\
CERNET & 1 & 3 \\
IBN31 & 3 & 3 \\
GEANT2 & 1 & 3 \\
\hline
\end{tabular}

reliabilities for all selected real-world networks and the node reliability of the corresponding Harary graph. We observed that, in all cases, $R(H, p) \geq R(G, p)$, pointing out that Harary graphs can represent a good topology in the sense of node reliability, and not only to the link reliability. The link reliability results were obtained from [20]. Comparing $R(G, p)$ and $R_{E}(G, p)$, notice that $R(G, p) \leq R_{E}(G, p)$ for all networks, and this fact may occur because a node failure affects one or more links although no mathematical proof has been done so far. Computing $S_{r}^{\prime}(G, p)$ and $S_{r}^{\prime}(H, p)$ for all

TABLE III: Link and node reliabilities results for the selected real-world networks, and node reliability of the corresponding Harary graphs

\begin{tabular}{cccc}
\hline Network & $R_{E}(G, p)$ & $R(G, p)$ & $R(H, p)$ \\
\hline CESNET & 0.987786 & 0.977832 & 0.998645 \\
ITALY & 0.996706 & 0.986030 & 0.999776 \\
NSFNET & 0.995581 & 0.993416 & 0.997940 \\
GARR-B & 0.993065 & 0.974732 & 0.998294 \\
GERMANY & 0.982903 & 0.965531 & 0.997271 \\
REDIRISNET & 0.981346 & 0.968150 & 0.998017 \\
EON & 0.992832 & 0.982579 & 0.998664 \\
COX & 0.987016 & 0.930437 & 0.996828 \\
CERNET & 0.974957 & 0.805304 & 0.994266 \\
IBN31 & 0.995861 & 0.992850 & 0.992850 \\
GEANT2 & 0.984636 & 0.835157 & 0.993563 \\
\hline
\end{tabular}

$r=1, \ldots, N$ is known for its high demanding computational time. For instance, computing the node reliability of the GEANT2 network spent about 72 hours. Even though we were able to compute it for all studied networks to understand how the number of connected induced subgraphs of $G$ e $H$ with $r$ nodes really influences the node reliability. Figures 3 and 4 together are important to understand such influence. In Figure 3 the number of nodes $r$ versus the number of connected induced subgraphs with $r$ nodes, $S_{r}^{\prime}(G)$, is displayed for the CESNET network. Note that in all cases, in particular when $r$ is close to $N, S_{r}^{\prime}(H) \geq S_{r}^{\prime}(G)$. The same situation occurs in the majority cases for other analysed networks, which is expected since in Table III we obtained $R(H, p) \geq R(G, p)$. Also, to understand the most relevant number of connected

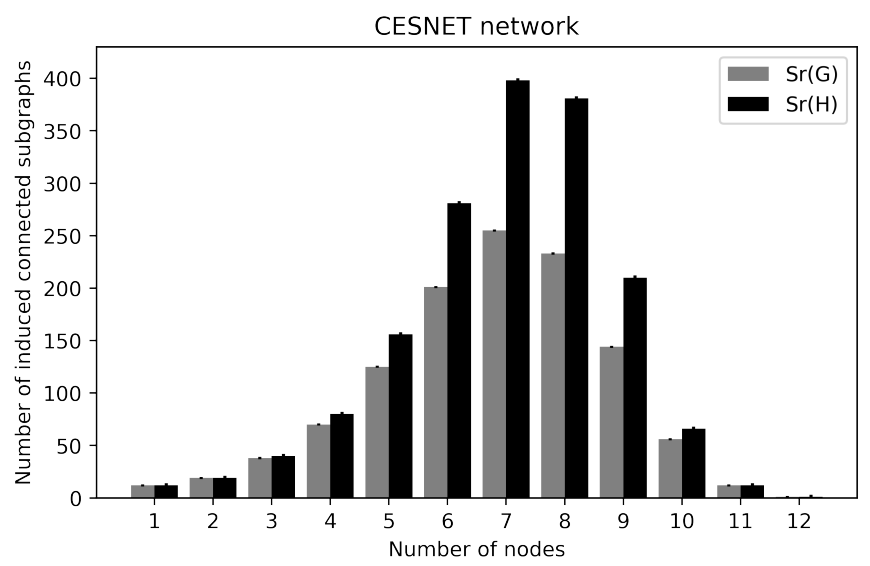

Fig. 3: Number of nodes $(r)$ versus Number of connected induced subgraphs with $r$ nodes $\left(S_{r}^{\prime}(G)\right)$ : CESNET network. In gray, $S_{r}^{\prime}(G)$, and in black, $S_{r}^{\prime}(H)$.

induced subgraphs of $G$ e $H$ with $r$ nodes in terms of influencing the node reliability, we compute the cumulative values of $R(G, p)$ as we obtain the number of node cut sets $S_{r}^{\prime}(G)$ for $r=1, \ldots, N$. The results are displayed in Figure 4 . It seems that the last terms of the function are most significant to the final computation of $R(G, p)$. Hence, we propose an approximation of the node reliability function considering only the last $N / 2$ final terms of Equation (1) as follows by Equation (3):

$$
R^{\prime}(G, p)=\sum_{r=\frac{N}{2}}^{N} S_{r}^{\prime}(G) p^{r}(1-p)^{N-r}
$$

In Table IV, we compute the percentual difference between the node reliabilities for each network using $p=0.05$ as follows:

$$
g a p=100\left(\frac{R(G, p)-R^{\prime}(G, p)}{R(G, p)}\right) .
$$

The results of Table IV show that the proposed function $R^{\prime}(G, p)$ is quite good for all eleven networks.

\section{CONCLUSION}

Designing a network in views of optimizing node reliability is desirable in several practical situations. The comparison of the node reliabilities of each of the eleven real-world networks with the node reliability of its corresponding Harary 
TABLE IV: Percentual difference between the reliability and the approximation of the node reliability function

\begin{tabular}{cc}
\hline Network & gap (\%) \\
\hline CESNET & $2.4 \mathrm{e}-4$ \\
ITALY & $7.6 \mathrm{e}-5$ \\
NSFNET & $4.1 \mathrm{e}-5$ \\
GARR-B & $5.3 \mathrm{e}-6$ \\
GERMANY & $2.3 \mathrm{e}-7$ \\
REDIRISNET & $5.8 \mathrm{e}-7$ \\
EON & $9.7 \mathrm{e}-8$ \\
COX & $2.3 \mathrm{e}-9$ \\
CERNET & $3.9 \mathrm{e}-12$ \\
IBN31 & 0.0 \\
GEANT2 & $4.52 \mathrm{e}-13$ \\
\hline
\end{tabular}

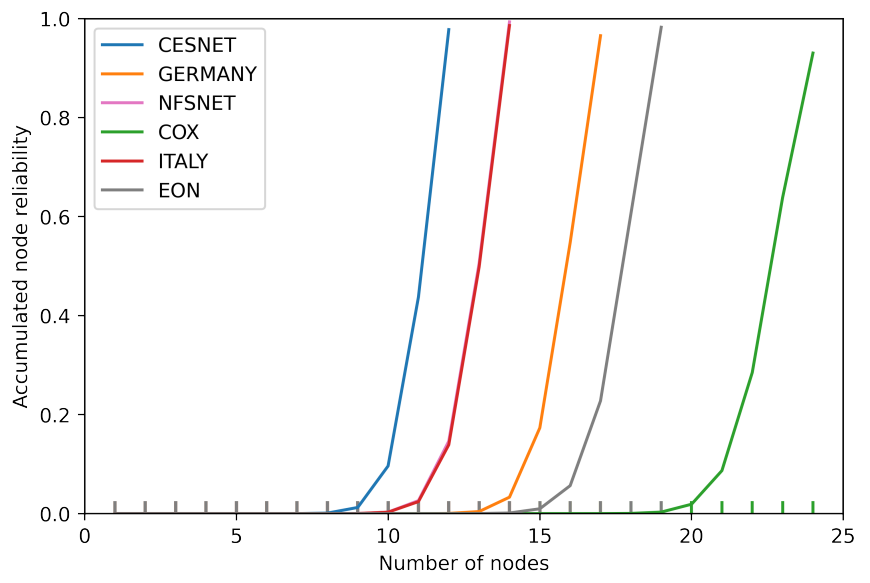

Fig. 4: Accumulated values of $R(G, p)$

graph has shown that, in all cases, the node reliability of a Harary graph is larger. Our results led us to conjecture that the Harary network topology is one of the most reliable topologies in terms of node reliability, besides having maximal link reliability as proved in the literature. Therefore, the Harary topology should be considered when designing a network with optimized node reliability. Further, we proposed a simplified formula that can be useful in networks with many nodes and links.

\section{REFERENCES}

[1] Bauer, D. and Boesch, F. and Suffel, C. and Tindell, R. "Combinatorial Optimization Problems in the Analysis and Design of Probabilistic Networks" in Networks, v. 15, pp. 257-271, 1985.

[2] Boesch, F. T., "On Unreliability Polynomials and Graph Connectivity in Reliable Network Synthesis," in Journal of Graph Theory, vol. 10, no. 3, pp. 339-352, 1986.

[3] Boesch, F. T. and Felzer, A., "On the Invulnerability of the Regular Complete k-Partite Graphs," in SIAM Journal on Applied Mathematics, vol. 20, pp. 176-182, 1971

[4] Cardoso, J. and Almeida, J. and Dias, J. and Coelho, P. "Structural Reliability Analysis Using Monte Carlo Simulation and Neural Networks" in Advances in Engineering Software, v. 39, n. 6, pp. 505-513, 2008.

[5] Dash, R. K. and Barpanda, N. K. and Tripathy, P. K. and Tripathy, C. R., "Network Reliability Optimization Problem of Interconnection Network Under Node-Edge Failure Model," in Applied Soft Computing, vol. 12, pp. 2322-2328, 2012.

[6] Deng, H. and Chen, J. and Li, R. "On the Construction of Most Reliable Networks", in Discrete Applied Mathematics, v. 140, pp. 19-33, 2004.

[7] Elshqeirat, B. and Soh, S. and Rai, S. and Lazarescu, M. "Topology Design with Minimal Cost Subject to Network Reliability Constraint" in IEEE Transactions on Reliability, v. 64, n. 1, pp. 118-131, 2015.
[8] Fishman, G. S. "A Monte Carlo Sampling Plan for Estimating Network Reliability" in Operations Research, v. 34, pp. 581-594, 1986.

[9] Goldshmidt, O. and Jaillet, P. and Lasota, R., "On Reliability of Graphs with Node Failures,", in Networks, vol. 24, pp. 251-259, 1994.

[10] Hakimi, S. L. , "An Algorithm for Construction of the Least Vulnerable Communication Network or the Graph with the Maximum Connectivity" in IEEE Transactions on Circuit Today, pp. 229-230, 1969.

[11] Harary, F., "Graph Theory" in Addison-Wesley Publishing Company, Massachusetts, 1969.

[12] Hössler, T. and Simsek, M. and Fettweis G. P., "Mission Reliability for URLLC in Wireless Networks" in IEEE Communications Letters, vol. 22, no. 11, pp. 2350-2353, 2018.

[13] Huang, J. and Feng, Y. and Zhang, S., "Research of Complex System Theory Application on Reliability Analysis of Network System," in 2009 8th International Conference on Reliability, Maintainability and Safety, 2009.

[14] Lee, K. and Lee, H. and Modiano, E. "Reliability in Layered Networks with Random Link Failures" in Proceedings of the 29th Conference on Information Communications (INFOCOM'10) in IEEE Press, Piscataway, NJ, USA, pp. 1667-1675, 2010.

[15] Li, Xiaowang and Zhou, Shuming and $\mathrm{Xu}$, Xiang and Lin, Limei and Wang, Dajin, "The Reliability Analysis Based on Subsystems of $(n, k)$ Star Graph," in IEEE Transactions on Reliability, vol. 65, no. 4, pp. $1700-1709,2016$

[16] Lima, L. S. and Teixeira, L. S. and Abreu, N. M. M., "Modelos de Redes Confiáveis," in Sistema \& Gestão, vol. 9, pp.488-494, 2014.

[17] Lima, L. S. and de Abreu, N. M. M. and Oliveira, C. S. and de Freitas, M. A. A., "Laplacian Integral Graphs in $\mathrm{S}(\mathrm{a}, \mathrm{b})$ " in Linear Algebra and Its Applications, vol. 423, pp. 136-145, 2007.

[18] Morales, F. G. and Paiva, M. H. M. and Bustos-Jiménez, J. A. "Measuring and Improving Network Robustness: A Chilean Case Study" in IEEE Communications Letters, pp. 44-47, 2019.

[19] Myrvold, W. and Cheung, K. H. and Page, L. B. and Perry, J. E., "Uniformly-Most Reliable Networks Do Not Always Exist" in Networks, pp. 417-419, 1991.

[20] Pavan, C. and de Lima, L. S. and Paiva, M. H. M. and Segatto, M. E. V., "How Reliable Are the Real-World Optical Transport Networks?," in J. Opt. Commun, vol. 7, no. 3, pp. 810-823, 2015.

[21] Shpungin, Y. "Combinatorial Approach to Reliability Evaluation of Network Unreliable Nodes and Unreliable links" in WASET 2006, 2006.

[22] Snow, A. "Network Reliability: the Concurrent Challenges of Innovation, Competition, and Complexity" in IEEE Transactions on Reliability, v. 50, n. 1 , pp. $38-40,2001$.

[23] The Internet Topology Zoo, The University of Adelaide, Australia [Online]. Available: http://www.topology-zoo.org/index.html, Accessed on: Dez. 25, 2018

[24] Yeh, M. S. and Lin, J. S. and Yeh, W. C. "A New Monte Carlo Method for Estimating Network Reliability" in Proceedings of the 16th International Conference on Computers \& Industrial Engineering, pp. 723-726, 1994.

[25] Yu, S. and Shao, F. and Meng, H. "Uniformily Optimal Graphs in Some Classes of Graphs with Node Failures" in Discrete Mathematics, v. 310 , n. 1, pp. 159-166, 2010. 